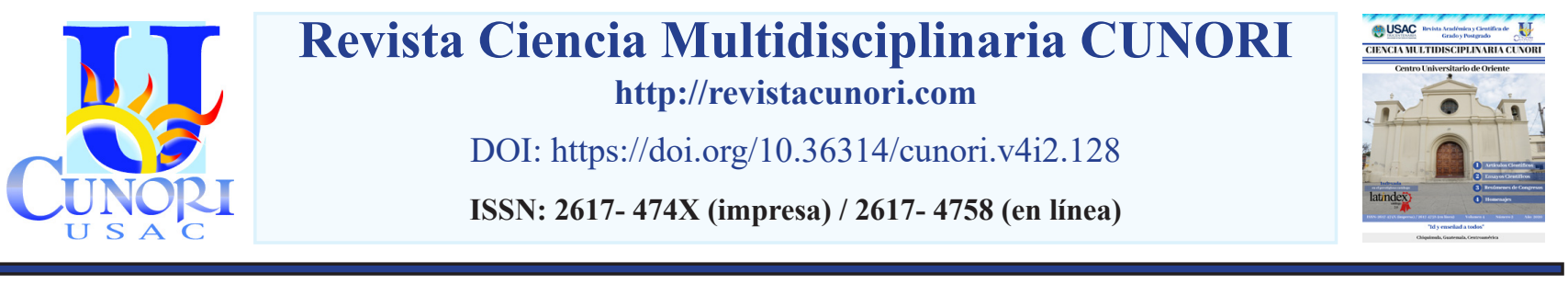

Referencia del artículo

Cruz-Barrera, B. M.; Martínez-Duarte, O. L.; Lucero-González, I. M.; Hernández, J. E. (2020). Caracterización de los estilos de aprendizaje en estudiantes de educación superior. Revista Ciencia Multidisciplinaria Cunori, 4(2), 61-67. https://doi.org/10.36314/cunori.v4i2.128

\title{
Caracterización de los estilos de aprendizaje en estudiantes de educación superior
}

\section{Characterization of learning styles in higher education students}

Brenda Maribel Cruz Barrera*,Olga Leticia Martínez Duarte, Ilcia Mireya Lucero González y Jesús Estuardo Hernández Doctorado en Investigación para el Desarrollo Social

Centro Universitario de Sur Oriente (CUNSURORI), Universidad de San Carlos de Guatemala

Recibido: 27 de mayo de 2020 / Revisado: 29 de junio de 2020 / Aceptado: 25 de septiembre de 2020

Disponible en internet el 30 de octubre de 2020

*Autor para correspondencia.

Correo electrónico: cruzbarrerabrenda@yahoo.es

Resumen

T a idea de escribir este artículo, nace del interés de desarrollar una investigación en el Centro Universitario de Sur Oriente, con

Uestudiantes de la Carrera de Administración de Empresas, con el objetivo principal de caracterizar los estilos de aprendizaje de los estudiantes que cursan la carrera; para tal efecto se consideró a una muestra de 204 estudiantes quienes estuvieron dispuestos a participar. Con los resultados alcanzados se pretende, sirva de soporte en la toma de decisiones de las autoridades y profesores que lideran y orientan el proceso de enseñanza en la institución, así como potencializar el desarrollo del aprendizaje y la generación de competencias profesionales en concordancia con la realidad cognitiva particular del estudiantado, para lo cual se utilizó el cuestionario CHAEA, de Alonso Gallego y Honey, de estilos de aprendizaje.

Palabras clave: aprendizaje, estilos, educación superior, caracterización

\footnotetext{
Abstract

$\mathrm{T}$ The idea of writing this article was born from the interest of developing an investigation in the South East University Center, with students of the Business Administration Career, with the main objective of characterizing the learning styles of the students who are studying the degree; For this purpose, a sample of 204 students who were willing to participate was considered. With the results achieved, it is intended to serve as support in the decision-making of the authorities and teachers who lead and guide the teaching process in the institution, as well as to enhance the development of learning and the generation of professional competencies in accordance with reality cognitive behavior of the student body, for which the CHAEA questionnaire, by Alonso Gallego and Honey, on learning styles was used.
}

Keywords: learning, styles, higher education, characterization 


\section{Introducción}

Desde la perspectiva técnica, la aplicación del cuestionario sobre estilos de aprendizaje en la educación superior, previo a iniciar un curso, puede constituirse en una herramienta diagnóstica para que el profesor conozca las distintas formas en que el grupo de estudiantes aprende y de qué forma el profesor puede orientar el aprendizaje, es decir planificarlo, seleccionar los métodos, técnicas, estrategias afines, para beneficiar al grupo en la adquisición del conocimiento, lo cual no es sencillo (Alonso,1994, pp. 60-61).

En la educación superior es importante que el docente sepa cómo tratar al estudiante adulto, desde el punto de vista de la neurociencia (Vallejo, 2016); es decir, saber el potencial de las personas luego de efectuar un diagnóstico individual, que conduzca a identificar el estilo de aprendizaje del grupo de estudiantes con los que interactúa en el aula. En virtud de lo cual, el docente tendrá una mejor visión del panorama y del ambiente motivador que puede generar en su entorno, así como contrarrestar el estrés que muchas veces se genera al momento de asignar tareas o actividades que en cierta forma provocan en el participante cansancio mental, físico e intelectual, cuando la metodología empleada no es acorde a sus necesidades, capacidades y/o habilidades.

Desde el punto de vista educativo, según Gutierres (2018, p.83), "el hecho de conocer la predominancia de los estilos de aprendizaje que tienen los participantes/estudiantes con los que se trabaja es fundamental para adaptar las metodologías docentes a las características que presentan los mismos, y así contribuir a elevar sus niveles de rendimiento educativo". Considerando los fundamentos descritos surge el interés de desarrollar una investigación en el Centro Universitario de Sur Oriente, con estudiantes de la Carrera de Administración de Empresas, con el objetivo principal de caracterizar los estilos de aprendizaje de los estudiantes que cursan la carrera; con el fin de que la información obtenida, conlleve a potencializar el desarrollo de las competencias profesionales en concordancia con la realidad cognitiva particular del estudiantado, y desarrolle de esta manera ventajas competitivas en el desempeño laboral actual, para lo cual se utilizó el cuestionario CHAEA, de Alonso Gallego y Honey, de estilos de aprendizaje.

\section{Materiales y métodos}

El estudio se desarrolló mediante el método de estudio de caso, a través de un diseño metodológico no experimental de tipo transversal. Entre las técnicas aplicadas estuvieron la observación y la encuesta. El instrumento manipulado para realizar la caracterización de los estilos de aprendizaje de los estudiantes de la Carrera de Administración de Empresas del Centro Universitario de Sur Oriente, de la Universidad de San Carlos de Guatemala, fue el cuestionario CHAEA, de estilos de aprendizaje que según Honey y Munford (1992) citados en De Armas y Rodríguez (2015, p. 18), se clasifican en cuatro estilos, estos son: Activo, reflexivo, teórico y pragmático. Esta catalogación se ha difundido y utilizado en las ciencias de la educación. 
Para el desarrollo investigativo, se trabajó con una población de 548 estudiantes inscritos formalmente, según la información proporcionada por control académico del CUNSURORI y se conformó una muestra de 204 estudiantes que atendieron la solicitud de proveer la información, de manera voluntaria. La investigación se desarrolló en los meses febrero a marzo, los ciclos que fueron abordados son: $1^{\circ}$., $3^{\circ} ., 5^{\circ} ., 7^{\circ}$ y $9^{\circ}$, que son los que se desarrollan normalmente durante el primer semestre del año.

\section{Resultados}

Los resultados de la investigación describen la caracterización de los estilos de aprendizaje de la población que fue objeto de estudio, que se conformó por una muestra de 204 estudiantes que colaboraron con la consecución del estudio de manera voluntaria. Para realizar el análisis de los resultados, identificar y caracterizar el estilo de aprendizaje de la población estudiada se utilizó el baremo del cuestionario donde se identifica la preferencia del estilo de aprendizaje individual con las categorías siguientes: Muy baja, baja, moderada, alta y muy alta, luego se realizó una tabla de frecuencias para determinar la preferencia de los estilos de aprendizaje por cada sujeto de estudio. La tabla 1 describe los resultados generales de acuerdo a preferencia de respuesta, también expresada en porcentaje.

Tabla No. 1 Estilos de aprendizaje de acuerdo a preferencia de respuesta y expresión en porcentaje.

\begin{tabular}{|c|c|c|c|c|c|c|c|c|c|c|c|}
\hline \multirow{2}{*}{$\begin{array}{l}\text { Estilos de aprendizaje } \\
\text { (categorías) }\end{array}$} & \multicolumn{2}{|c|}{ Muy baja \% } & \multicolumn{2}{|c|}{ Baja \% } & \multicolumn{2}{|c|}{ Moderada \% } & \multicolumn{2}{|c|}{ Alta \% } & \multicolumn{2}{|c|}{ Muy alta \% } & \multirow{2}{*}{$\begin{array}{c}\text { Total \% } \\
100\end{array}$} \\
\hline & 17 & 8.33 & 33 & 16.18 & 73 & 35.8 & 34 & 16.67 & 47 & $\begin{array}{c}23 . \\
03\end{array}$ & \\
\hline Reflexivo & 24 & 11.8 & 29 & 14.21 & 55 & 26.96 & 37 & 18.13 & 14 & $\begin{array}{c}6.8 \\
6\end{array}$ & 100 \\
\hline Teórico & 10 & 4.90 & 22 & 10.8 & 100 & 49 & 47 & 23.03 & 70 & $\begin{array}{c}34 . \\
31\end{array}$ & 100 \\
\hline Pragmático & 21 & 10.3 & 21 & 10.3 & 61 & 29.90 & 48 & 23.52 & 53 & $\begin{array}{c}25 . \\
98\end{array}$ & 100 \\
\hline
\end{tabular}

Cruz-Barrera, B. M.; Martínez-Duarte, O. L.; Lucero-González, I. M.; Hernández, J. E. (2020). Información de la base de datos del trabajo de campo.

La información que se expresa en la tabla refleja que el estilo teórico es el que presenta la mayor cantidad de estudiantes (100), representada por el $49 \%$ de la población objeto de estudio, que muestran preferencia por esta forma de aprender, pero es de considerar que si bien, es el que más estudiantes de la muestra lo eligen, lo hacen en una forma moderada, pues es la categoría moderada la de mayor ponderación, en los cuatro estilos de aprendizaje. Así también, se muestra que 73 estudiantes se inclinan por el estilo activo; en tercer lugar, el estilo pragmático, con el cual se identifican 61 estudiantes y por último el estilo reflexivo, que muestran una preferencia hacia este, 55 estudiantes. Es importante hacer notar que los cuatro estilos se presentan en una forma moderada en la población estudiada. 


\section{Discusión}

El estudio presenta varias fortalezas porque se conoce el estilo de aprendizaje predominante de los estudiantes objeto de la investigación, así mismo que los alumnos participaron voluntariamente, lo que a su vez fue una limitante porque no se obtuvo el cien por ciento de la intervención del estudiantado. En el centro universitario a nivel de posgrado se realizó un estudio en 2018 en relación a los estilos de aprendizaje, que fue aplicado a estudiantes de plan domingo de la carrera de pedagogía en donde se obtiene como resultado que los cuatro estilos de aprendizaje (activo, teórico, reflexivo y pragmático) que predominaron en la población estudiada, lo que contrasta con el estudio realizado en la carrera de Administración de Empresas en el primer semestre del 2020, tomando en consideración que es otra carrera, otro grupo de estudiantes y las características del individuo.

Cada vez es necesario profundizar en la manera en que aprenden los estudiantes y al hacerlo se logra caracterizar los estilos de aprendizaje de la población estudiantil, en este caso, que cursa la Carrera de Administración de Empresas y los resultados denotan que prefieren hacerlo de forma teórica, esto se debe a que siempre espera las orientaciones que contribuyen en asegurar de forma conductual el aprendizaje, no cabe duda que esto motiva en la enseñanza, pero cuando el estudiante recibe de forma teórica los procedimientos de las tareas, con recursos de apoyo, encuentra coherencia en el desarrollo de sus actividades de aprendizaje, además asume con más preferencia esta forma de aprender, no obstante tales características de estilo de aprendizaje sobresaliente pueden ir modificándose conforme avance en la carrera y las experiencias con el entorno.

En la medida que los estudiantes avanzan, es importante que se adapten al contenido de una forma más activa, reflexiva y pragmática, para que logren procesar el conocimiento de manera diversa y no únicamente en un estilo que se trunca o depende exclusivamente de lo teórico, debido a que en esta área del conocimiento, los estudiantes se preparan no solo para seguir instrucciones, sino para tomar decisiones en ambientes donde no siempre se tiene el apoyo teórico, al contrario el aprendizaje se construye en función del contexto y la realidad que se vive, lo cual debe generar y crear a partir del desarrollo de nuevas ideas que le permitan desenvolverse mejor, con seguridad y confianza desde la libertad de sus propios intereses, además el estudiante debe descubrir y conocer desde sus propias experiencias, en donde la teoría y la práctica se complementan para generar competencias que contribuyen al avance de nuevos estilos de aprendizaje que fortalecen el desarrollo académico y la libertad de actuar con decisión propia.

Los resultados indican que cada vez es necesario profundizar en la manera en cómo aprenden los estudiantes porque de esta forma se les puede beneficiar al seleccionar la metodología idónea y planificar las actividades de aprendizaje que conlleven a promover el desarrollo de las competencias profesionales tomando como referencia la realidad cognitiva que presenta cada uno. Los resultados de este estudio pueden variar dependiendo de las experiencias estudiantiles, laborales y del contexto que vayan experimentado los mismos, además de los grupos de estudiantes que ingresen a la carrera, pero pueden ser tomados en cuenta para otros estudios sobre los estilos de aprendizaje y a la vez se sugiere realizarlos para hacer comparaciones y que surjan nuevos temas de investigación. 
Hay que tener claro que profesores y estudiantes aprenden de diferentes formas, para el profesor no será tarea fácil adaptarse al estilo de aprendizaje de cada estudiante, principalmente si tiene un grupo muy diverso, pero sí, debe hacer el esfuerzo por favorecer al alumnado en general al logro de las competencias a desarrollar como parte del curso, diseñando actividades variadas que se ajusten a las características de cada forma de aprender, sin olvidar las inteligencias múltiples, dando opciones para realizar la misma actividad de evaluación de distinta manera, considerando que en cada individuo se percibe una forma característica de aprender (Honey y Mumford, 1992 citados en de Armas y Rodríguez, 2015).

Los estudiantes participantes en el estudio, mostraron tendencia hacia los estilos de aprendizaje activo, reflexivo, teórico y pragmático, en una categoría moderada, sin embargo, los resultados evidencian que se aprende de forma diferente, lo que se constituye en un desafío para los profesores de la carrera porque tienen que capacitarse sobre los diversos métodos, técnicas y estrategias de enseñanza aprendizaje a emplear de acuerdo a la forma en que adquieren el conocimiento y planificar actividades que favorezcan el desarrollo de las capacidades y respondan a las maneras de aprender de la población estudiada, de los diversos ciclos para favorecer a todos por igual y orientar hacia el aprendizaje significativo.

Los resultados del cuestionario de estilos de aprendizaje son una herramienta eficiente, para medir de qué manera el estudiante prefiere afrentar el nuevo reto de aprender, en donde lo activo, reflexivo y pragmático es moderado y lo teórico resulta ser sobresaliente, en estilo y nivel de aprendizaje, ante esta información cuantitativa, es un reto grande para los educadores universitarios de la Carrera de Administración de Empresas, el romper paradigmas cuando los estudiantes creen que aprenden mejor, cuando se concentran en lo teórico, dependerá de los procesos de planificación de los docentes, para ir generando estrategias de aprendizaje donde los estudiantes descubran estilos de aprendizaje que los preparen para la vida, con mente abierta a los cambios abruptos que la modernidad presenta, porque al desarrollar nuevos estilos de aprendizaje se conducirá a la mejora significativa.

\section{Agradecimientos}

A la comunidad académica de la carrera de Administración de Empresas del Centro Universitario de Sur Oriente de la Universidad de San Carlos de Guatemala.

\section{Referencias}

Alonso, G. (1994). Los Estilos de Aprendizaje, procedimientos de diagnóstico y mejora. Bilboa : Mensajero.

De Armas, M. y Rodríguez, M. (2015). "Estudio de los estilos de aprendizaje de estudiantes y profesores de la Facultad de Ingeniería de la Universidad Central de Venezuela”. Revista de la Facultad de Ingeniería U.C.V. Vol. 30, No. 3, pp. 17-26.

Gutierres, M. (2018). Estilos de Aprendizaje, estrategias para enseñar. Su relación Su relación con el desarrollo emocional y "aprender a aprender". Tendencias Pedagógicas. No 31 doi: http://dx.doi. org/10.15366/tp2018.31.004, 83-96. 
Vallejo, D. (2016). "Neurociencia en la Educación superior: desde el enfoque de la transdisciplinariedad y estilos de aprendizaje”. Revista científica INVESTIGAR. Segunda edición. Pp 21-32.

\section{Sobre los autores}

\section{Brenda Maribel Cruz Barrera}

Es Licenciada en Administración de Empresas, es Maestra en Ciencias en Educación y Ambientalización Curricular. Actualmente se encuentra en proceso de graduación en el Programa de Doctorado en Investigación para el Desarrollo Social. Es asesora de proyectos de investigación a nivel técnico, Licenciatura y Postgrado en Maestrías. Se desempeña como profesora del CUNSURORI, colabora en las revisiones de artículos para graduación de Maestría y ha publicado en revistas científicas a nivel nacional.

\section{Olga Leticia Martínez Duarte}

Es Licenciada en Pedagogía y Administración Educativa se graduó en la Maestría en Educación con especialidad en Docencia Universitaria. Está en proceso de graduación en el Doctorado en Investigación para el Desarrollo Social. Es profesora Interina de la carrera de Pedagogía del CUNSURORI -USACTambién ha sido profesora de Postgrado de la Universidad Mariano Gálvez de Guatemala y del CUNSURORI. Realizó una investigación sobre la relación entre la metodología de aprendizaje participativo y el fomento del pensamiento crítico en los estudiantes del nivel superior. El estudio lo realizó en las Facultad de Humanidades y Derecho del Centro Universitario de Jalapa, de la Universidad Mariano Gálvez de Guatemala.

\section{Ilcia Mireya Lucero González}

Es Licenciada en Administración de Empresas. Posee una Maestría en educación y ambientalización curricular. Es profesora titular I de la Carrera de Administración de Empresas en el Centro Universitario de Sur Oriente CUNSURORI de la USAC. Es asesora de proyectos de investigación a nivel técnico y licenciatura. Ha trabajado como profesora en la Escuela de Postgrado del CUNSURORI.

\section{Jesús Estuardo Hernández}

Es docente del CUNSURORI - JALAPA se desempeña como consultor de Fhi360, USAID y de Juárez y Asociados en programas educativos. Colabora como revisor de artículos de investigación, además de publicar en la revista Científica, Educación y Sociedad, de la Universidad de Ciego de Ávila de Cuba el tema "Experiencias del acompañamiento pedagógico en escuelas rurales de Jalapa y Mataquescuintla". 
Copyright (c) Brenda Maribel Cruz Barrera*,Olga Leticia Martínez Duarte, Ilcia Mireya Lucero González y Jesús Estuardo Hernández

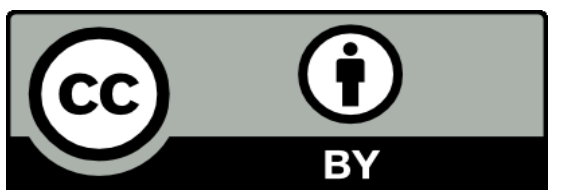

Este texto está protegido por una licencia CreativeCommons 4.0.

Usted es libre para compartir, copiar y redistribuir el material en cualquier medio o formato y adaptar el documento, remezclar, transformar y crear a partir del material para cualquier propósito, incluso comercialmente, siempre que cumpla la condición de atribución: usted debe reconocer el crédito de una obra de manera adecuada, proporcionar un enlace a la licencia, e indicar si se han realizado cambios. Puede hacerlo en cualquier forma razonable, pero no de forma tal que sugiera que tiene el apoyo del licenciante o lo recibe por el uso que hace. 